\title{
TARRADELLAS, EL ORGANIZADOR. LA CAMPAÑA PROPAGANDÍSTICA DEL ESTATUTO DE AUTONOMÍA DE CATALUÑA DE 1932
}

\author{
JOAN ESCULIES SERRAT \\ GRENS - Universitat Pompeu Fabra \\ jesculies@hotmail.com
}

(Recepción: 23/09/2015; Revisión: 22/01/2016; Aceptación: 14/04/2016; Publicación: 02/12/2016)

\begin{abstract}
1. INTRODUCCIÓN.-2. LA APARICIÓN DE UN JOVEN POLÍTICO.-3. EL PUBLICISTA: PLEBISCITO Y REFERÉNDUM.-4. El RELACIONES PÚBLICAS: VIAJE A MADRID.-5. El ESCENÓGRAFO: AZAÑA EN BARCELONA.-6. LA TRASLACIÓN DE LA EXPERIENCIA PROFESIONAL A LA POLÍTICA.-7. BIBLIOGRAFÍA
\end{abstract}

\begin{abstract}
RESUMEN
El artículo explora un aspecto desconocido de la biografía de Josep Tarradellas: su papel como organizador de la campaña propagandística del Estatuto de autonomía de Cataluña de 1932. Se analiza su rol de publicista, relaciones públicas y escenógrafo a partir de la traslación a la política de las habilidades aprendidas en su profesión, viajante de comercio. El texto muestra las claves que permitieron a Tarradellas convertirse en una figura imprescindible para el gobierno provisional de la Generalitat de Catalunya de Francesc Macià en el periodo 1931-1932 pese a ser un recién llegado a la política.
\end{abstract}

Palabras clave: Tarradellas; Macià; Esquerra Republicana de Catalunya; Estatuto de Autonomía; Azaña. 


\title{
TARRADELLAS, THE FIXER. THE PROPAGANDA CAMPAIGN OF THE CATALAN STATUTE OF AUTONOMY IN 1932
}

\begin{abstract}
The paper explores an unknown aspect of the biography of JosepTarradellas: his role as the fixer of the propaganda campaign of the Catalan Statute of Autonomy in 1932. It is discussed Tarradellas' role as publicist, public relations and scenographer pointing out how he used the skills learned as a salesman into political tools. The research shows the factors that allowed Tarradellasto become a key figure for the provisional government of the Generalitat de Catalunya, led byFrancescMacià, in the period 1931-1932 despite being a newcomer to politics.
\end{abstract}

Keywords: Tarradellas; Macià; Esquerra Republicana de Catalunya; Catalan Statute of Autonomy; Azaña.

$$
* \quad * \quad *
$$

\section{INTRODUCCIÓN}

Josep Tarradellas, presidente de la Generalitat de Catalunya (1954-1980), es una de las figuras políticas española más citadas -sobre todo por lo que se refiere a su papel durante la Transición- pero todavía existen notables lagunas en cuanto a la investigación de su extensa trayectoria (1). El siguiente artículo se propone cubrir un aspecto concreto de su biografía: su papel como organizador de la campaña propagandística del Estatuto de autonomía de Cataluña de 1932. Se usa aquí el concepto de «campaña» en su sentido más amplio comprendiendo la organización del plebiscito de los ayuntamientos catalanes y el referéndum para la aprobación del anteproyecto de Estatuto, el viaje del entonces presidente del gobierno provisional de la Generalitat de Catalunya, Francesc Macià, a Madrid para entregarlo a Cortes y el viaje a Barcelona del presidente del consejo de ministros, Manuel Azaña, una vez aprobado el texto. El artículo se plantea como un caso de estudio para comprender mejor la actuación de Tarradellas en unos años en que el joven catalanista probó sus aptitudes como político y extrajo unas primeras lecciones que le resultaron muy útiles durante los años de la Guerra Civil española y exilio, cuando desplegósus capacidades con toda su amplitud (2).

(1) Algunas aproximaciones a su biografía: IVERN (1988, 1989); AlQUÉZAR (2001); ARBós (1988); Bonamusa (2008); Bricall (1977); CASASSAS (2003); CONTE (2011).

(2) Tarradellas $(1998,1999,2007)$; Soler Serrano (1977); Sobrequés (1988); SEnTís (2002). 


\section{LA APARICIÓN DE UN JOVEN POLÍTICO}

Sin duda alguna, si un nombre apareció de manera fulgurante en el panorama político de la Cataluña republicana de los años treinta este fue el de Josep Tarradellas (1899-1988). Con treinta y dos años y una trayectoria política previa prácticamente inexistente, su figura pasó de ser desconocida a imprescindible para los suyos, esto es para Esquerra Republicana de Catalunya (ERC) (3). «Terra» o «Tarra», como le denominaban sus allegados, asomó en la política catalana al comenzar el año 1931, con su participación en la refundación de la Joventut Nacionalista La Falç [Juventud Nacionalista La Hoz]. La entidad había sido una de las más destacadas de entre la docena que en el periodo 19181923 conformaron el nacionalismo radical catalán -el primer separatismo organizado- en Barcelona (4).

La ideología de este ultracatalanismo, por otra parte muy minoritario, se asentaba sobre tres pilares básicos. En primer lugar, un separatismo poco concreto con un espectro que abarcaba desde el autonomismo, la federación o confederación en un Estado español o ibérico, al independentismo. A esto se sumaba una posición antielectoralista que los jóvenes intransigentes habían heredado de los postulados iniciales del nacionalismo catalán y de la primera gran plataforma transversal, Unió Catalanista, a partir de la cual habían aparecido las entidades ultracatalanistas. Finalmente, la ideología intransigente se completaba con un posicionamiento socializante hacia los segmentos obreros. Es decir, los trabajadores de «cuello blanco», que eminentemente formaban las entidades del nacionalismo radical catalán, tenían una cierta comprensión, una mirada paternal, hacia los trabajadores de «cuello azul» (5).

La mayoría de estos dependientes «saltataulells» u oficinistas «pixatinters», se habían formado en las escuelas del Centre Autonomista de Dependents del Comerç i de la Indústria (CADCI) barcelonés (6). Precisamente en el CADCI fue donde Tarradellas recibió su formación y conoció a los compañeros que le llevarían a afiliarse a La Falç, más para buscar un espacio de socialización, para conocer chicas y bailar sardanas, que no persiguiendo ningún interés político. En una entidad como La Falç, de entre sus doscientos socios, apenas el grupo que formaba su consejo directivo estaba politizado. Tarradellas cursó cuatro años de estudios de las escuelas del Centro a partir de1914, cuando había llegado a Barcelona con su familia procedente de Cervelló, en la actual comarca del Baix Llobregat, a escasos treinta quilómetros al sur de la capital catalana (7).
(3) Esculies (2012b).
(4) EsCULIES $(2013,2014)$.
(5) UCElay-da CAL (1987): 183-193.
(6) Lladonosa (1988).
(7) Esculies (2012). 
En el CADCI siguió el currículo que formaban a los dependientes, vendedores y viajantes de comercio, que constaba de cursos de geografía comercial, aritmética, nociones de álgebra y un idioma, en el primer año; economía y cálculo comercial, entre otros, en el segundo; técnica de los negocios, redacción de documentos, correspondencia y transportes, en el tercero; y la especialización concluía con un cuarto año que incidía en legislación aduanera, publicidad moderna y química. Asimismo, allí aprendió francés y algo de inglés (8). Mientras se formaba, Tarradellas ejerció de dependiente en una tienda de tejidos.

Su paso por Melilla como personalsanitario durante el servicio militar poco después del «desastre de Annual», durante año y medio, le acabó de convencer, como a tantos otros jóvenes como él, que la Monarquía era una vía muerta. A su retorno y ante el anticatalanismo de la dictadura de Primo de Rivera, el joven afianzó su convicción de que la salida política para Cataluña, pero también para el resto de España, se hallaba en la consecución de la República (9). A pesar de ello, a su regreso a Barcelona, Tarradellas no participó en actividades clandestinas y, por el contrario, se propuso no ejercer más como dependiente y progresar como viajante de comercio por cuenta propia.

A partir del invierno de 1923, el joven emprendedor o self-mademan planteó una estrategia simple para obtener clientes: identificó diversas áreas fabricantes de tejidos, cristalería, porcelana y productos de caucho en Europa y en los Estados Unidos, contactó con cerca de ochenta compañías entre 1923 y 1934 y obtuvo la representación -a comisión, no como agente comprador- para toda España de seis de ellas, de las que se encargaba al mismo tiempo. De este modo, Tarradellas consiguió una situación económica envidiable que le permitió en adelante no depender de la política en cuanto finalmente decidió dedicarse a ella (10).

Caído Primo, pues, y en el marco de la Dictablanda, «Tarra» se reunió con sus compañeros de La Falç, algunos de los cuales la habían mantenido en hibernación con el nombre de Humorístic Club, y se involucró en la refundación de la entidad dispuesto, ahora sí, a entrar en la liza política. Por su pasado por el nacionalismo radical catalán y puesto que Macià había sido nombrado presidente honorario de La Falç en 1919 -a pesar de no haber podido atraer esta entidad a sus intereses políticos al comienzo de los años veinte debido al antielectoralismo de sus asociados-, ahora la opción lógica de Tarradellas y La Falç fue participar en la denominada Conferència de les Esquerres Catalanes (11).

La Conferencia era una suerte de foro de debate, los días 17 y 19 de marzo de 1931, entre republicanos catalanes aunados al entorno de Lluís Companys y Marcel-lí Domingo, grupos republicanos catalanistas de comarcas como el Foment Nacionalista Republicà de Reus de Josep Andreu Abelló, el grupo con

\footnotetext{
(8) Febrés (1985): 11; DuRAN (2003): 35; PorCel (1977): 12, 17; Udina (1978): 31, 35-36.

(9) RoIG (1992).

(10) EsCULIES (2012).

(11) Albert (1939).
} 
aires socialdemócratas editor del periódico L'Opinió, con Joan Lluhí Vallescà y Joan Casanelles al frente, y por supuesto los nacionalistas radicales de Estat Català de Macià y Jaume Aiguader, junto a los grupos intransigentes pre-dictatoriales refundados como La Falç. El foro a la postre daría lugar al nacimiento del partido político, Esquerra Republicana de Catalunya (12).

Fue en el marco de esa Conferencia, mientras todo el mundo hablaba y daba órdenes sobre lo que convenía y lo que no, pero pocos ejecutaban, cuando «Tarra» tomó papel y lápiz y comenzó a ordenar la agenda de Francesc Macià, apabullado ante tanta demanda para visitar centros y ateneos catalanistas un mes escaso después de su regreso de un exilio de siete años. Fue ese gesto, sin más, lo que le convirtió en secretario particular de facto del Avi (el abuelo), el líder del nuevo partido (13).

Ante las elecciones municipales del 12 de abril de 1931, Macià encomendó a su secretario, pese a no figurar en las candidaturas, la misión de organizar la campaña, principalmente en Barcelona. Como recordaría Casanelles, «Tarradellas quería solucionar todos nuestros problemas a través de sus fichas» (14). Y, aunque por supuesto la victoria sorprendente -por inesperada- de ERC en los comicios, sobre todo en Barcelona capital, se debió más a la excepcionalidad de la situación que a la gestión de los mítines, sin duda el hecho que «Tarra» estructurara la participación de los cuadros de un partido recién nacido contribuyó a ella.

Después de la proclamación de la República, primero española, por Lluís Companys y luego catalana, por Macià, la tarde del 14 de abril, de nuevo fue Tarradellas quien puso orden junto al capitán Eduardo Medrano en el Palacio de la Diputación de Barcelona: su altura, 1,80 m, le ayudó a imponer su criterio. Fue «Tarra» quien se encargó de permitir el paso a los periodistas cuando estos se veían impedidos para entrar por compañeros suyos que para nada comprendían que las acciones políticas tenían tanto de gesticulación como de propaganda. Él, en cambio, siempre tuvo muy claro que para ganarse al receptor de sus mensajes en primer lugar era necesario ganarse al transmisor (15).

El 17 de abril, ya con la República catalana transformada en el gobierno provisional de la Generalitat de Catalunya, para cuando Macià le nombró secretario particular de iure, la capacidad organizativa de Tarradellas había quedado totalmente demostrada. Tanto, que incluso Joan Lluhí Vallescà y el republicano Joan Casanovas se disputarían años después quién de ellos había recomendado al Avi la elección de «Tarra» para el puesto (16). De esta manera, después de organizar la secretaría del presidente de la Generalitat y su oficina de prensa, en junio de 1931 se encargó de dirigir la campaña de las elecciones a Cortes

(12) Culla (1981); UCELAY-da CAL (1977).

(13) EsCULIES (2015); ARRUfat (2007): 300, 309-310.

(14) CASANELLES (1991): 92, 114.

(15) Llates (1969): 552; PALAu (1932).

(16) Lluhí (1932); Casanovas (1996): 99-100. 
Constituyentes, en la que resultó elegido diputado, y en julio creó la oficina de la Generalitat en Madrid, una suerte de delegación del gobierno provisional catalán y nexo de unión entre los parlamentarios catalanes y la cámara (17).

En apenas un par de meses desde su aparición en el momento fundacional de ERC, Tarradellas se convirtió en imprescindible para Macià. Era el «fixer» (el solucionador de problemas) del presidente. Lo mismo que el joven Stalin lo había sido para Lenin en los albores de su revolución, aunque salvando, claro está, las distancias ideológicas y sin tener que caer en tejemanejes ilegales para obtener recursos. La razón era idéntica a la que había expuesto el líder bolchevique para justificar la elección del georgiano: «Se le podía asignar cualquier trabajo con la certeza que quedaría bien hecho» (18). Para un hombre como el $A v i$, de setenta y dos años, tener al lado una garantía como la que representaba «Tarra» era sin duda un hallazgo.

Tarradellas no era un buen orador. Él mismo asumía que no destacó en su período como diputado en Cortes (1931-33), ni tampoco en el Parlamento catalán a partir de 1932 por esa razón. No contaba con el carisma de Macià, ni tampoco con el verbo fácil de Companys. En la Cataluña populista que cuajaba en los años treinta su figura como cabeza de cartel difícilmente habría arrastrado electorado suficiente para ganar nada (19). Y, sin embargo, el primer año y medio de la Generalitat provisional de Macià difícilmente se puede entender sin su labor entre bambalinas.

Lo que hizo que se convirtiera en una figura estratégica fue la traslación de su experiencia profesional a la organización de la política. Tarradellas demostró que su formación, aunque elemental, bien aplicada podía dar mucho de sí. Durante los años de la dictadura había conseguido una cómoda situación económica, pero sobre todo había desarrollado su habilidad para clasificar clientes y tareas mediante su archivo -las «fichas» a que se refería Casanelles- y entrenó, por así decirlo, su don de gentes, que tan útil le resultó en su trato con la prensa, con la que vivió un verdadero idilio, especialmente en 1931 y 1932.

\section{EL PUBLICISTA: PLEBISCITO Y REFERÉNDUM}

Ante el comienzo de la andadura de la Segunda República, la consecución del Estatuto de autonomía de Cataluña se convirtió en el objetivo principal de la legislatura para el grupo de la Minoría catalana en Cortes, encabezado por ERC, su partido mayoritario. Una vez redactado el anteproyecto estatutario a finales de junio de 1931 en el Hotel del Valle de Núria, en el Pirineo catalán, este fue aprobado por la asamblea de la Diputación provisional y la Generalitat
(17) GonZalez (2003).
(18) Sebag (2007): 332.
(19) UCELAY-da CAL (1982). 
fijó la celebración de un plebiscito entre los ayuntamientos para el 26 de julio y de un referéndum entre la población para el 2 de agosto. La organización jurídica de ambos fue encargada al entonces consejero adjunto, abogado e íntimo de Macià, Amadeu Hurtado (20).

El sábado 18 de julio se anunció la constitución de la oficina de propaganda del Estatuto (21). Tarradellas acababa de regresar de Madrid donde había inaugurado, junto a Companys y otros diputados, la oficina de la Generalitat en la capital de la República, y una vez más, Macià le puso al frente de la situación. De este modo -y a pesar que quizás lo lógico hubiese sido encomendar la misión al consejero de Gobernación, Casanovas, a su entorno o a alguien con una mayor trayectoria política y edad-, el nombramiento de «Tarra» vino a instaurar un método de funcionamiento que sería recurrente en el periodo de la Generalitat provisional de Macià. Este consistía enencargar a Hurtado la parte jurídica de las situaciones -la Generalitat «hacia dentro», por así llamarlo- y a Tarradellas la proyección pública de estas -la Generalitat «hacia fuera»-.

De este modo, la oficina de propaganda del Estatuto quedó instalada en el Palacio de la Generalitat-el antiguo Palacio de la Diputación y sede del gobierno- el lunes 20 de julio, dotándola de personal y material necesario para resolver cualquier duda que ofreciese la aprobación del anteproyecto y con la voluntad «de promover una activísima campaña para despertar la más firme adhesión en todas las capas sociales de Cataluña» (22). Durante su primer día de actividad, la oficina recibió la visita de numerosos secretarios de ayuntamientos. Asimismo, se enviaron quince mil circulares a diferentes entidades, organizaciones y administraciones de Cataluña «manifestando la importancia del momento político actual y aconsejando la más activa propaganda a favor de la ley particular», junto con ejemplares del manifiesto que Macià había hecho público el día anterior pidiendo el voto favorable al proyecto.

Tarradellas acompañó a los periodistas que cubrían la información de la Generalitat por las dependencias de la oficina. Su director y secretario particular del presidente les informó que ésta redactaría y repartiría copias de toda la documentación referente al plebiscito de los ayuntamientos para que cada uno recibiese en un sobre un ejemplar del Estatuto, una carta firmada por Macià y un modelo de acuerdo municipal, que únicamente sería necesario firmar y sellar caso que el

(20) IVERN (1988): 132; ROIG (1978); HURTADO (2011): 634-635.

(21) «Josep Tarradellas a l'aparell», L'Opinió, 16 de julio de 1931, p. 1; «La propaganda de l'Estatut de Catalunya», Ibid., 16 de julio de 1931, p. 5; «L'Estatut de Catalunya», Ibid.,18 de julio de 1931, p. 4; Estatuto de Cataluña», La Vanguardia, 18 de julio de 1931, p. 6; «La propaganda de l'Estatut», La Publicitat, 16 de julio de 1931, p. 2; El nombre de la oficina no aparece en el Butlletí Oficial de la Generalitat de Catalunya. En ocasiones recibió el de Oficina ProEstatutó Oficina d'Informació de l'Estatut. Hemos optado por el de Propaganda por considerar que es el más adecuado a su finalidad.

(22) «Un manifiesto del presidente/La organización del referéndum», La Vanguardia, 19 de julio de 1931, p. 6; «Manifest de Macià», L'Opinió, 19 de julio de 1931,p. 1. 
consistorio correspondiente acordara la adhesión al proyecto. Al finalizar la visita, Tarradellas ofreció un cóctel a los periodistas. Al día siguiente estos en sus crónicas destacaban que en la oficina «se vivía una actividad febril» (23).

Durante la semana del lunes 20 al domingo 26 de julio, la labor de la oficina se centró en facilitar información a los ayuntamientos y en recibir las adhesiones de estos. Según la prensa, «la actividad en estos días ha sido formidable, se han ciclostilado 140.000 hojas» (24). Tarradellas quiso destacar también que, además de la preparación del plebiscito y el referéndum, la oficina se dedicaba a facilitar a todos los interesados los datos, informaciones y ejemplares necesarios del Estatuto, tanto si se trataba de una entidad como de un particular. La oficina estaba abierta cada día al público de doce a dos y de cinco a ocho de la tarde.

A partir del miércoles fue necesario aumentar el personal, que rondaba ya el centenar de personas. Se atendían desde comunicaciones relativas a la organización del referéndum a telegramas y actos dando cuenta de la aprobación del Estatuto por parte de los municipios catalanes o a delegados de todo tipo de entidades que pedían directrices para organizar mítines u otros actos de propaganda. A finales de semana, su director recibió los primeros elogios desde el vocero de ERC, L'Opinió: «No podemos más que felicitar al señor Tarradellas por los grandes dotes como organizador que ha mostrado tener con la instalación de la oficina de propaganda» (25).

Una vez finalizada la fase plebiscitaria de los ayuntamientos, con una aprobación del proyecto de Estatuto casi unánime, a partir del lunes 27 y hasta el domingo 2 de agosto, la labor de la oficina entró en el periodo de máxima actividad y se focalizó en la propaganda dirigida a la ciudadanía. A tal efecto, se habilitaron, además de las dependencias que desde el primer día se habían reservado, el salón de sesiones y cinco salones más del Palacio de la Generalitat.

Tarradellas mandó que entre el martes y el miércoles quedasen fijados en Barcelona veinticinco mil carteles y que en cada una de las entradas a la ciudad se dispusieran grandes lonas con lemas que manifestaban la importancia del referéndum. En la semana y media de actividad, los pedidos de la Generalitat a nueve imprentas barcelonesas consumieron treinta toneladas de papel. Se imprimieron cinco millones de papeletas para la votación, setenta y cinco mil carteles de diferentes colores y textos, siete millones de boletines de propaganda con párrafos del mensaje del presidente a la Diputación provisional y la respuesta de esta.

(23) «Oficina de Información», La Vanguardia, 21 de julio de 1931, p. 8; «Unes oficines al servei del públic», La Publicitat, 21 de julio de 1931, p. 3.

(24) «Una oficina model», L'Opinió, 22 de julio de 1931, p. 2.

(25) «Per a la campanya pro Estatut de Catalunya», La Publicitat, 22 de julio de 1931, p. 2 «Una fase de gran activitat a les oficines de Pro-Estatut», Ibid., 23 de julio de 1931, p. 2; «Campanya de propaganda», La Vanguardia, 23 de julio de 1931, p. 5; «Una fase de gran activitat a les oficines pro-estatut de la Generalitat», L'Opinió, 24 de julio de 1931, p. 3. 
También se confeccionaron banderolas para los coches del servicio público, carteles con la imagen de Macià pidiendo el voto favorable, ediciones especiales de artículos periodísticos recomendando la votación plebiscitaria y se repartieron en todos los cines de Cataluña diferentes clichés para proyectar durante las sesiones hasta el domingo (26). La oficina también editó hojas oficiales para recoger adhesiones femeninas, ya que estas no podían votar (27). Se fijaron carteles en las fachadas de los edificios, en los árboles y escaparates. Los ferrocarriles de las líneas Madrid-Zaragoza-Alicante y del Norte, los trenes eléctricos de Barcelona-Sabadell y los tranvías llevaban carteles en los vagones y locomotoras. Asimismo se celebraron numerosas conferencias y mítines para dar a conocer el anteproyecto y recomendar el voto afirmativo.

En medio de la intensa actividad desplegada, L'Opinió elogió de nuevo a Tarradellas y tranquilizó a los que temían que la campaña propagandística no era lo suficientemente intensa (28). Una sensación que, a tenor de lo expuesto, solo unos pocos podían tener. Incluso la tarde del viernes 30, Macià y su hombre de confianza y consejero de Instrucción Pública, Ventura Gassol, se desplazaron al aeródromo Canudas para presenciar la salida de cuatro aeroplanos que sobrevolaron el cielo de Barcelona con la bandera catalana pintada bajo las alas, lanzando miles de panfletos de propaganda. Al día siguiente, los pilotos recorrieron Cataluña entera repartiendo publicidad.

El sábado por la tarde también se celebró, a modo de clausura de los actos propagandísticos, una caravana automovilística que recorrió la Avenida 14 de abril -la actual Diagonal-, para después pasar por los barrios de Sarriá, Gracia y acabar en la Generalitat. Según destacó La Vanguardia, la campaña llegó «a proporciones que no encuentran comparación con ningún otro acto político de los realizados en Barcelona y Cataluña» (29). También el periodista Andreu-Avelí Artís, quien más tarde sería conocido por el pseudónimo «Sempronio», consideraba desde Mirador que se había batido el récord de propaganda política (30).

A primeras horas del domingo 2 de agosto, la oficina reunió todo el personal acreditado para ayudar en lo necesario a la constitución de las mesas electorales y no retardar el proceso. También allí se instaló una estación emisora de radio

(26) «El president de la Generalitat», L'Opinió, 24 de julio de 1931,p. 1; «Mensaje del señor Macià», La Vanguardia, 24 de julio de 1931, p. 25; «Las oficinas de propaganda», Ibid., 28 de julio de 1931, p. 7; «La participación femenina», Ibid., 29 de julio de 1931,p. 4; Mirador, 30 de julio de 1931, p. 5; «Avís important relacionatamb el referèndum del dia 2 d'agost», La Publicitat, 29 de julio de 1931, p. 2; «Intensa activitat a les oficines», Ibid., 31 de julio de 1931,p. 2.

(27) GonZaLez (2006); MARTín (2013).

(28) «Suggestions, propaganda...», L'Opinió, 31 de julio de 1931, p. 1.

(29) La Vanguardia, «Propaganda aérea», 29 de julio de 1931, p. 4; «La Campaña», Ibid., 2 de agosto de 1931,p. 6; «Actos de propaganda en favor del Estatuto», Ibid., 26 de julio de 1931, p. 22; «Per l'Estatut de Catalunya», L'Opinió, 23 de julio de 1931, p. 2; «Propaganda aèria», Ibid., 29 de julio de 1931, p. 2; «Aeròdrom Canudes», La Publicitat, 1 de agosto de 1931, p. 9; «Una gran mobilització cívica», Ibid., 2 de agosto de 1931, p. 5.

(30) Andreu-Avelí Artís, «Anècdotes i fervors de l'Estatut», Mirador, 6 de agosto de 1931, p. 2. 
para dar a conocer las últimas novedades en relación al referéndum. Durante la mañana pasaron por el Palacio los consejeros del gobierno catalán y algunos diputados. A las cuatro de la tarde se presentó Macià. El Avi, como hecho anecdótico y pese a la persistente campaña amenazante contra aquellos que no acudiesen a las urnas, no votó en el referéndum porque estaba censado en Lérida, y no quiso desplazarse hasta allí (31).

A media tarde, Andreu-Avelí Artís se presentó también en las dependencias de la oficina de propaganda, encontrándolas casi desiertas y con su director completamente agotado. «Tarradellas, el generalísimo del Estatuto -narró el reportero-, esperaba los primeros resultados de la batalla repanchingado en una silla. Después de una semana febril, ahora le había entrado también esa lasitud que enmudece a los teléfonos y paraliza a las máquinas de escribir» (32). La labor de la oficina se dio por finalizada a las once de la noche, con su director dando todavía las últimas órdenes. El referéndum contó con una participación del $75 \%$ del censo catalán, cerca de ochocientas mil personas, un $99 \%$ de las cuales votaron afirmativamente (33).

\section{EL RELACIONES PÚBLICAS: VIAJE A MADRID}

Una vez aprobados el plebiscito y el referéndum, Tarradellas, ayudado por los efectivos de la oficina de Madrid, se encargó de organizar el viaje de Macià a la capital de la República para entregar el anteproyecto de Estatuto al gobierno español y a Cortes (34). En esta ocasión su tarea se centró en cuadrar la agenda del presidente, en cuanto a visitas y a entrevistas, en un perfil entre secretario y relaciones públicas. No fue requerida ahora su participación en organizar eventos, más allá de conseguir que ningún contratiempo desmereciese la visita y, como siempre, que los periodistas catalanes desplazados para la ocasión estuviesen en todo momento correctamente atendidos. Una vez más quien acompañó a Macià en las reuniones de trabajo fue Hurtado.

Además de su secretario particular y los consejeros Ventura Gassol, Manel Carrasco i Formiguera y Pere Comas, el alcalde de Barcelona, Jaume Aiguader y esposa, los diputados Joan Puig i Ferreter, Josep Riera i Puntí, Antoni M. Sbert, Josep Selvas, Nicolau Battestini y Josep A. Trabal, Macià se hizo acompañar por su hija Maria. Esta hacía las veces de primera dama catalana puesto que la esposa del Avi, Eugenia Lamarca, aborrecía la política -como también la esposa de Tarradellas, Antònia Macià (quien pese a su apellido no guardaba

(31) «En la Generalidad», La Vanguardia, 4 de agosto de 1931, p. 8.

(32) «Anècdotes i fervors de l'Estatut», Mirador, 6 de agosto de 1931, p. 2.

(33) «A la Generalitat», La Publicitat, 4 de agosto de 1931, p. 1.

(34) AYMAMí (1932). 
parentesco alguno con el presidente) - y se dejaría ver en contadas ocasiones a su lado en actos públicos (35).

La comitiva salió de la estación de Francia de Barcelona en el expreso vespertino del 13 de agosto de 1931. El gentío que fue a despedir a Macià era tan considerable que ocupaba el vestíbulo de la estación y los andenes y, en el exterior, el basto espacio entre el Palacio del Gobierno civil y el Parque de la Ciudadela. La aglomeración era tal que se suspendió la salida de otros trenes hasta que lo hubiese hecho el expreso del presidente. Tarradellas llegó a las ocho menos cuarto junto a Macià y Comas. El Avi se vio impotente para sortear el gentío y llegar a su vagón y tuvo que subir al tren por el de cola. Desde allí se dirigió a los congregados para expresar que viajaba a Madrid para entregar el Estatuto «con los brazos abiertos». Hasta el último instante Tarradellas, calificado por L'Opinió como «el hombre impasible que ha dado pruebas de ser un gran político», se ocupó que ningún contratiempo entorpeciese la despedida (36).

El expreso de Macià llegó a la estación de Atocha al mediodía siguiente. Allí fue recibido por, entre otras personalidades, los ministros catalanes, Marcel·lí Domingo de Instrucción pública y Lluís Nicolau d'Olwer de Economía. Tarradellas comunicó por teléfono al consejero de Gobernación, Joan Casanovas, que ejercía de presidente de la Generalitat y de alcalde de Barcelona accidental, que el recibimiento en la capital española había sido equiparable a la despedida en Barcelona. En la misma línea se expresaba el enviado especial de L'Opinió, Joan Alavedra, socio de La Falç, amigo de «Tarra» (37).

Una visión más cáustica de la llegada la dio Rafael Font i Farran, también desde L'Opinió, expresando que debido a la tensión ambiental de los últimos días el recibimiento se podía considerar un éxito, pese a que buena parte de los allí congregados eran catalanes (38). Esta era, a tenor del recelo con que viajaban los catalanes a Madrid, una versión más acorde con lo sucedido. Incluso Alavedra había confesado al corresponsal de La Veu de Catalunya en Madrid, Josep Pla, que viajaba con una pistola en el bolsillo por si las moscas (39). Es decir, el periodista del vocero de ERC, que a la postre substituiría a «Tarra» como secretario particular de Macià, ejercía, cabe suponer que junto a otros miembros de la expedición, como guardaespaldas a la sombra.

En esta misma línea, El Noticiero Universal publicó que a su paso por la Puerta de Atocha Tarradellas había sacado una pistola ante una posible agresión

(35) SARDÀ (1989): 220-227; VIUSÀ (1968): 97-98.

(36) «Francesc Macià a Madrid», L'Opinió, 13 de agosto de 1931, p. 1; «Una gentada imponenta comiadàahir Francesc Macià i els parlamentaris catalans», Ibid., 14 de agosto de 1931, p. 1; «La partida del presidente de la Generalitat», La Vanguardia, 14 de agosto de 1931, p. 4. p. 6.

(37) «El presidente accidental de la Generalidad», La Vanguardia, 15 de agosto de 1931,

(38) «El viatge del president de la Generalitat de Catalunya», L'Opinió, 16 de agosto de 1931, p. 4.

(39) Pla (1996): 101. 
a Macià. Incluso desde el periódico satírico catalán El Be Negre, se explicaba en clave de humor que había sacado una «pipa [un evidente juego de palabras] del bolsillo, aunque no fumase». Un hecho que en principio puede parecer inverosímil y que él mismo desmintió, pero que dado lo comentado por Alavedra, no es descartable (40). Y es que los ánimos ante el Estatuto comenzaban a exaltarse y no dejarían de hacerlo durante toda la tramitación parlamentaria.

La comitiva catalana se alojó en las habitaciones de la 470 a la 476 de la cuarta planta del Hotel Palace, en el ala izquierda del edificio con vistas a la plaza Cánovas. A continuación asistieron al banquete en el Ritz, ofrecido por Niceto Alcalá-Zamora (41). Una vez finalizado el almuerzo, a las cuatro, Unión Radio Madrid entrevistó a Macià y Aiguader y, al finalizar, estos se dirigieron a la presidencia del gobierno provisional de la República. Allí se habían dispuesto un par de micrófonos para radiar los discursos de los dos presidentes en el marco de la ceremonia de entrega del anteproyecto de Estatuto (42).

A las seis de la tarde, el presidente catalán fue al Congreso, donde aprovechó para prometer el acta de diputado, cosa que no había hecho desde su elección, el 28 de junio. Saludó al presidente de las Cortes, Julián Besteiro, y recibió una ovación de pie de los diputados -excepto de Miguel de Unamuno y Antonio Royo Villanova, que además permanecieron sentados-. A continuación Macià siguió la sesión desde su escaño, entre Amadeu Hurtado y Jaume Carner. Lo mismo hizo Tarradellas desde el suyo.

La estancia de Macià en Madrid se alargó más de lo previsto porque su hija María no había visitado la ciudad, y el presidente quiso combinar encuentros protocolarios y reuniones de trabajo con visitas turísticas. Por otra parte, Macià y Alcalá-Zamora mantenían un trato cordial ya desde la etapa pre-dictatorial, cuando habían coincidido como diputados en Cortes. María y las hijas del cordobés, Fura y María Teresa, también tuvieron buen entendimiento, lo que no añadió excesiva prisa al mandatario catalanista para acabar con su visita (43).

Entre el sábado 15 y el martes 18, Tarradellas se encargó de la organización de las visitas turísticas de Macià y su séquito, incluyendo a los periodistas catalanes. Durante estos días el grupo visitó el Escorial, Ávila, Aranjuez -pudieron visitar las habitaciones del Hotel Comercio donde había muerto el pintor catalán Santiago Rusiñol-, Toledo -con visita al Alcázar y a la parroquia de San Vicente para admirar los Grecos y la iglesia de Santo Tomás para ver El entierro

(40) «Una informació de 'El Noticiero' falsa», L'Opinió, 15 de agosto de 1931; «Manifestaciones del alcalde», La Vanguardia, 16 de agosto de 1931, p. 4; «Conversa amb Companys», El Dia, 17 de agosto de 1931, p. 1; «La veritat sobre l'afer Tarradellas», El Be Negre, 18 de agosto de 1931, p. 2.

(41) «L'arribada a Madrid», La Publicitat, 15 de agosto de 1931, p. 1.

(42) «El president de la Generalitat de Catalunya a Madrid», L'Opinió, 15 de agosto de 1931, pp. 1-4; «En el Congreso», La Vanguardia, 15 de agosto de 1931, p. 18; AlCALÁ-ZAMORA (2012): 322-323; PEÑA (2002): 157-160.

(43) GabRIEL (2000). 
del conde Orgaz-, la casa museo del Greco en Santa María la Blanca, San Juan de los Reyes y Los Cigarrales.

Macià cenó también con el ministro de la Guerra, Manuel Azaña, en Navacerrada, donde este descansaba y con el que el Avi distaba mucho de tener la relación cordial que mantenía con don Niceto. En Madrid visitó a Alcalá-Zamora en su domicilio particular y aprovechó para encontrarse con su hermana monja, que ejercía de superiora en un convento y a la que hacía diecisiete años que no había visto. También visitó la tumba de Goya en San Antonio de La Florida, entreteniéndose un buen rato contemplando los aguafuertes de la sacristía $D e$ sastres de la Guerra.

Macià, para agradar a su hija, invitó a las hijas de Alcalá-Zamora a almorzar en el Palace, mientras en el Hotel Savoy se celebraba un banquete de conmemoración del Pacto de San Sebastián. Por su parte, Tarradellas ejerció, como de costumbre, de portavoz, y comentó en diversas ocasiones a los periodistas catalanes que el presidente estaba muy satisfecho con las visitas. También, en una práctica de uso habitual en su actuación como relaciones públicas, organizó un cóctel en el café Gran Vía para unos ochenta catalanes que se habían desplazado a la capital española en motivo de la entrega del Estatuto (44).

Los siguientes días, del miércoles 19 al sábado 22, tuvieron más carga política. Tarradellas organizó la agenda del presidente y convocó a los asistentes catalanes a las sesiones de trabajo. Macià presidió un par de reuniones de la Minoría catalana y junto a Hurtado trató con el ministro de la Guerra del acuartelamiento de tropas en Cataluña. También, siempre con Hurtado, se entrevistó con los ministros de Estado, Alejandro Lerroux, de Fomento, Álvaro de Albornoz, de Hacienda, Indalecio Prieto, y de Justicia, Fernando de los Ríos. Por otra parte, Macià no asistió, deliberadamente, a la sesión de presentación del anteproyecto de Estatuto en Cortes. El catalán no quería quedar en evidencia al tener que responder a la intervención de Alcalá-Zamora, puesto que no era un hábil orador como el cordobés (45).

Finalmente, a las diez menos cuarto de la mañana del domingo 23, después de nueve días en Madrid, Macià, Tarradellas y el resto de la comitiva tomaron el expreso de vuelta a Barcelona. En el andén les despidieron Alcalá-Zamora y sus hijas, Rafael Sánchez-Guerra y Nicolau d'Olwer, entre otros. El séquito

(44) «Excursión de los representantes de Catalunya», La Vanguardia, 16 de agosto de 1931, p. 15; «Excursiones a Aranjuez y Toledo», Ibid., 18 de agosto de 1931, p. 18; «Ahir el president de la Generalitat visità l'Escorial i Àvila. Per tot fouacollitamb gran estima», L'Opinió, 16 de agosto de 1931, p. 1; «L'estada del president de la Generalitat a Madrid», Ibid., 18 de agosto de 1931, p. 4.

(45) «Visitas del señor Macià», La Vanguardia, 20 de agosto de 1931, p. 20; «El ministro de Guerra», Ibid., 20 de agosto de 1931, p. 15; «Manifestaciones del señor Macià», Ibid., 21 de agosto de 1931, p. 15; «El viat getriomfal d'en Macià», L'Opinió, 19 de agosto de 1931, p. 1; «L'estada del president de la Generalitat a Madrid», Ibid., 19 de agosto de 1931, p. 5; «L'estada de Francesc Macià a Madrid», Ibid., 22 de agosto de 1931, p. 4; «L'acció dels diputats catalans», Ibid., 22 de agosto de 1931, p. 4. 
llegó a Barcelona casi a medianoche y, de nuevo, la estación de Francia se llenó de gente. El auto que llevó a Macià a la Generalitat tardó muchísimo en llegar. Y una vez allí este tuvo que descansar unos minutos, mientras Carrasco i Formiguera hacía las veces de telonero, antes que el Avi pudiera dirigir unas palabras a sus conciudadanos desde el balcón del Palacio de la Generalitat, donde se habían instalado micros para radiar su discurso (46).

Después del viaje, La Publicitat, órgano del opositor de centro-izquierda, Partit Catalanista Republicà, remarcó que Tarradellas había recibido a la prensa «vencido por la enorme labor que estos días ha pesado sobre él» (47).

\section{El ESCENÓGRAFO: AZAÑa EN BARCELONA}

Un año después de la presentación del anteproyecto de Estatuto en Cortes y habiendo sobrevivido a una tramitación harto difícil, el 15 de septiembre de 1932, Josep Tarradellas asistió en San Sebastián a la ceremonia de firma del Estatuto de autonomía de Cataluña por parte del ahora presidente de la República, Alcalá-Zamora. De regreso a Barcelona inició los preparativos para la visita del presidente del Consejo de ministros, Manuel Azaña, y del presidente de las Cortes, Julián Besteiro, a Cataluña, entre el domingo 25 y martes 27 de septiembre, con motivo de la aprobación del texto.

A sabiendas de su capacidad organizativa la oposición no tuvo ningún reparo, más bien al contrario, en que la responsabilidad de los preparativosdel viaje de Azaña recayeran en Tarradellas, que había dejado de ser secretario particular de Macià en abril por voluntad propia, y que desde diciembre de 1931 ocupaba el cargo de consejero de Gobernación. Por supuesto, quienes más tranquilos estaban con «Tarra» llevando las riendas del asunto eran los cuadros de la propia Esquerra. La Humanitat, periódico dirigido por Companys aparecido en noviembre de 1931 como vocero del partido, se felicitaba que fuera él el elegido porque «es una garantía de que las cosas se organizarán bien» (48). El joven consejero, en otra muestra del saber hacer que le caracterizaba, tuvo en todo momento informados al capitán general de Cataluña, Domènec Batet, y a los cuatro gobernadores civiles catalanes, con quienes mantuvo diversas reuniones para darles a conocer y comentar el programa que preparaba.

En el momento de organizar los actos, y sobre todo de la llegada de la comitiva encabezada por Azaña y Besteiro, Tarradellas todavía tenía muy frescas las aglomeraciones que se habían producido un año atrás en la estación de Francia con motivo del viaje de ida y vuelta de Macià a Madrid. El joven

(46) «Salida del Sr. Macià para Barcelona», La Vanguardia, 25 de agosto de 1931, p. 19; «Después de la entrega del Estatuto de Cataluña», Ibid., 25 de agosto de 1931, p. 6.

(47) «El senyor Tarradellas», La Publicitat, 25 de agosto de 1931, p. 2.

(48) «Del meu carnet», La Humanitat, 22 de septiembre de 1932, p. 1. 
consejero no quiso que la situación se repitiera y que el ejecutivo catalán proyectara una impresión de desbarajuste. Una impresión que pudiera afectar el traspaso de competencias y, en concreto, la de orden público al pensar que se podía transferir a un gobierno que la reclamaba pero que no la podría mantener.

Tarradellas, como buen viajante de comercio, era consciente de que a la hora de convencer o agradar no importa lo que uno es, sino cómo se presenta o representa ante los demás. En su cargo como secretario particular había acompañado a Macià en sus incontables visitas por las poblaciones catalanas y había visto durante un año y medio muy de cerca el valor de la imagen del presidente entusiasmando a sus conciudadanos sin pronunciar palabra (49). Partiendo de esta experiencia planteó un recibimiento que Azaña no pudo olvidar.

Para evitar un desorden de multitudes, Tarradellas mandó acreditar al personal que creía que debía tener acceso al interior de la estación de Francia y en el Palacio de la Generalitat. Y, siempre atento con la prensa, dispuso que en él se habilitara una dependencia especial para los medios de comunicación locales y los desplazados desde la capital española. Durante la semana pidió en diversas ocasiones a través de la prensa que para recibir a Azaña la ciudadanía no se amontonase en la estación, sino que se dispusiera a lo largo del recorrido hasta la Generalitat. También indicó que las entidades y corporaciones que asistieran con la bandera respectiva debían situarse a lo largo de Vía Layetana entre la plaza de Antonio López y la del Ángel. Incluso, puesto que la plaza de la República -hoy San Jaime- no tenía cabida para todos, Tarradellas dispuso altavoces en las calles colindantes para que se pudieran seguir los discursos de los presidentes desde el balcón del Palacio.

Además, ordenó pintar unas rayas blancas en la plaza de la República para señalar los espacios que podía ocupar la gente y los que debían de quedar libres para que pasara la comitiva. También pidió que las diversas comisiones no fueran a la Generalitat hasta las doce, hora prevista de llegada de Azaña, para evitar esperar en balde. En la recepción del Palacio solo podían asistir los ayuntamientos y las corporaciones políticas, económicas, culturales y obreras, representadas como máximo por cuatro personas. El día 23 mandó marchar hacia Reus a personal de la Generalitat para hacerse cargo de todo el equipaje de los expedicionarios. De este modo, al salir de la recepción e ir a los hoteles respectivos, las maletas ya estarían en su sitio. El consejero de Gobernación dispuso asimismo que se diera a cada visitante una cartera con las invitaciones necesarias para su estancia en Barcelona, además de pases para los trenes, tranvías, taxis, funiculares, buses, teatros, cines y parque de atracciones.

La prensa destacaba que Tarradellas no dejaba un instante de trabajar en la organización de la recepción de Azaña. Llegó incluso a autorizar a un representante de la Paramount para filmar con aparatos sonoros procedentes de París la llegada del presidente del Consejo. El 24 de septiembre por la tarde, el ministro

(49) UCELAY-dA CAL (1984). 
de Gobernación, Santiago Casares Quiroga decidió sumarse a la expedición. En cambio, Besteiro anunció que no iría a Barcelona debido a una gastralgia (50).

Finalmente, el día 25 a las diez de la mañana, Tarradellas salió de Palacio con los consejeros Gassol, Casanovas y Josep Jové. Minutos después en otro automóvil lo hizo Macià con su hija Maria. En la estación de Francia aguardaba el servicio de vigilancia del cuerpo de carabineros con bayoneta calada y banderas de la República y catalanas. Una compañía del regimiento de infantería número 10 también estaba presente para rendir honores a Azaña. Otra compañía, la del regimiento de infantería número 34, le recibiría a la puerta del Hotel Colón, donde debía alojarse.

La locomotora que traía a Azaña iba adornada con un escudo de Cataluña, lazadas catalanas y flores naturales. «Tarra» había mandado al escenógrafo, Josep Rocarol, a Zaragoza para decorarla. A su llegada, Azaña pasó revista mientras, pese a las medidas, algunas personas rompían el cordón policial y conseguían subir a los vagones del expreso. A pesar de lo previsto costó también salir del vestíbulo de la estación porque la gente quería saludar a los presidentes (51). Aun así, la comitiva subió a los automóviles en el patio de la estación bajo la dirección del joven consejero.

En los primeros autos iban los concejales, seguidos de los diputados por la Generalitat y a Cortes y los miembros del consejo ejecutivo. Seguían los coches de Aiguader, Casares Quiroga y el jefe de la Minoría catalana, Companys. Siendo el último para Azaña y Macià. Cerraba una sección de caballería de la Guardia Urbana con uniforme de gala y el regimiento de caballería número 10 que, a medida que la comitiva pasaba, se iba replegando (52).

Una de las cosas que más llamó la atención fue la disposición que diseñó Tarradellas de tres círculos concéntricos de guardias de asalto formando una cadena que rodeaba el coche presidencial. El satírico Defensa dels Interessos Catalans (D.I.C.) llegó a sugerirle que «otra vez que vuelvan, quedará mucho mejor, disponiendo que de los tres grupos de guardias que bailaban la sardana, uno monte una pilar de castellers [pilares humanos] y otro dance el ball de gitanes [baile con cintas], o bien el de capsgrossos [cabezudos]». Más allá de la

(50) «La estancia del señor Azaña en Barcelona», La Vanguardia, 20 de septiembre de 1932, p. 6; «El homenaje de Cataluña», Ibid., 25 de septiembre de 1932, p. 8; «La entrega del Estatuto. Viaje del señor Azaña», Ibid., 24 de septiembre de 1932, p. 6; «A la Generalitat. L'ordre», La Veu de Catalunya, 26 de septiembre de 1932, p. 2; «El viatge del cap de govern a Barcelona», L'Opinió, 23 de septiembre de 1932, p. 7; «Madrid-Barcelona amb el primer estadista español», La Rambla, 26 de septiembre de 1932, p. 5.

(51) Rocarol (1999).

(52) «La visita del Consejo y de las Cortes», La Vanguardia, 22 de septiembre de 1932, p. 4; «El viaje del jefe de Gobierno», Ibid., 27 de septiembre de 1932,p. 8; «Ornamentació de la màquina», La Veu de Catalunya, 23 de septiembre de 1932, p. 2; «La formació de la comitiva», L'Opinió, 27 de septiembre de 1932, p. 2; «Catalunya reptriomfalment els homes liberals d'Espanya»,La Rambla, 26 de septiembre de 1932, pp. 1-2. 
ironía, los comentarios unánimes a la organización del recibimiento de Azaña fueron del todo positivos (53).

Para organizar el trayecto a la Generalitat, Tarradellas se inspiró en las ticker tape parade estadounidenses. Estos desfiles, normalmente celebrados en Nueva York, se iniciaron de manera espontánea en 1886 con la inauguración de la estatua de la Libertad y tienen lugar para recibir una celebridad, bien sea un presidente o al personaje público que haya conseguido alguna hazaña. El desfile recibe el nombre de ticker tape porque se lanzan grandes cantidades de papel picado desde los edificios altos, en la Quinta Avenida, por ejemplo, por donde pasa el coche descubierto con el homenajeado.

La disposición de los guardias alrededor del vehículo, Tarradellas la copió de estos desfiles. Los más recientes en Nueva York antes de la recepción de Azaña en Barcelona habían sido los de Amelia Earhart, tras su vuelta transatlántico en junio de 1932, la del mariscal Pétain en octubre de 1931, la de los atletas olímpicos estadounidenses en agosto de 1928 o la de junio de 1927 al aviador Charles Lindbergh, después de su vuelo transatlántico, que impactó en especial al joven organizador.

El efecto que este mimetismo -sin el papel picado, claro está- provocó en Azaña fue grande y al encarar Vía Layetana y ver las banderas a ambos lados, y el gentío, expresó «caramba, qué barbaridad» (54). Una manifestación notable en un hombre que pasaba por tener un temperamento contenido. Azaña, llegó a asegurar que, «si en mi partido tuviese una docena de muchachos con el espíritu político y el entusiasmo y la voluntad, trabajo y la fe de Tarradellas, otra sería la vida política de estas Cortes y otro el camino venturoso de la República» (55). Unas declaraciones que nada tendrían que ver con su percepción de la actuación de «Tarra» durante la Guerra Civil (56).

Al llegar a la plaza del Ángel, Azaña se levantó para contemplar Vía Layetana una vez más. A continuación, la estrecha calle de Jaime I, que conduce a la plaza de la República, donde de otro modo se hubiese podido producir un tapón monumental, no supuso problema alguno puesto que Tarradellas había prohibido apostarse en ella para facilitar así el paso de la comitiva. Pese el gentío de la plaza había un cordón de la guardia municipal y los serenos vestidos de gala, que facilitó no solo el desplazamiento de la comitiva, sino también el de la prensa, con lo que la impresión de los periodistas fue totalmente positiva. El trayecto de la estación hasta el Palacio duró una hora.

A pesar del éxito organizativo, Macià no guardaba la misma relación con Azaña que con Alcalá-Zamora y durante la visita los presidentes coincidieron

(53) «Amb Azaña per Barcelona», La Rambla, 26 de septiembre de 1932, p. 3.

(54) «Que és gran Barcelona!», Mirador, 29 de septiembre de 1932, p. 1; Nevius (2009): 275-277; JACKSON (2010).

(55) POBLET (1976): 113.

(56) AZAÑA (2007): 386. 
lo mínimo. Nada más aparecer en el balcón del Palacio, Macià se dirigió a la multitud para asegurar que la República se había conseguido sin derramar ni una gota de sangre. Una imagen que desagradó al de Alcalá de Henares y a raíz de ello pidió ver el discurso que haría el catalán por la noche en el banquete que se le ofrecía en la Lonja. Pese a que el Avi accedió a ello y el gabinete de Azaña introdujo numerosas modificaciones, al final se optó por no dar parlamentos. Por la tarde, Azaña asistió a una recepción ofrecida por Batet, a la fiesta organizada en Montjuic y, después del banquete, a la representación de Terra Baixa [Tierra Baja] de Margarita Xirgu y Enric Borràs en el Teatro Olympia, con Macià (57).

Al día siguiente ninguno de los dos presidentes fue a la excursión a Núria como estaba previsto. Azaña alegó un resfriado y se quedó en Barcelona para presidir la reunión del comité regional de su partido, Acción Republicana, y a mediodía visitó Montserrat acompañado del gobernador Moles, pero de ningún consejero (58). Tarradellas, por su parte, participó en el homenaje a José Salmerón, en el restaurante El Parc de la Ciutadella. Por la noche, asistió al banquete de homenaje de la Asociación de Periodistas de Barcelona al periodista madrileño Luis Bello, sentándose junto a él en la presidencia en el restaurante Miramar. Mientras tanto, Macià cenaba con Azaña, Aiguader, Gassol y esposas en el Palacio Nacional de Montjuic y luego visitaban el Pueblo Español.

Esa misma noche, el joven consejero de Gobernación acompañó a Joan Moles, gobernador civil de Barcelona, a la estación de Francia para despedir a Casares Quiroga, y al día siguiente acompañó a Azaña en su visita a Lérida con Bello, Companys y también Moles. Allí visitaron la Paeria (el ayuntamiento), los Campos Elíseos, la Catedral vieja y el Castillo del Gardeny. Macià alegó encontrarse cansado y se quedó en Barcelona. Por la noche, en el Hotel Londres de Reus, el presidente catalán, deseoso ya que la visita acabase, ofreció un banquete de despedida a Azaña (59).

(57) «La recepció», L'Opinió, 27 de septiembre de 1932, p. 3; «L'Hotel Colom», Ibid., 27 de septiembre de 1932, p. 6; «El cap del Govern de la República a Barcelona», La Veu de Catalunya, 27 de septiembre de 1932,p. 3; «L'Àpat de l'Associació de Periodistes de Barcelona a Luís Bello», Ibid., 27 de septiembre de 1932, p. 7; «El senyor Azaña va sortircap a Lleida», Ibid., 28 de septiembre de 1932, p. 3; «Manifestacions del senyor Tarradellas», Ibid., 30 de septiembre de 1932, p. 2

(58) Rivas (1961).

(59) «El viatge del senyor Azaña a Barcelona», La Publicitat, 23 de septiembre de 1932, p. 1; «El president del Consell i el ministre de Governació a Montserrat», Ibid., 27 de septiembre de 1932,p. 4; «L'homenatge a Josep Salmeron», Ibid., 27 de septiembre de 1932, p. 4; «El diad'ahir a la Generalitat», Ibid., 27 de septiembre de 1932, p. 6; «Excursión a Montserrat-Excursión a Núria», Ibid., 27 de septiembre de 1932, p. 12; «El cap del Govern de la República a Barcelona», Ibid., 27 de septiembre de 1932, p. 3; «La visita del senyor Azaña», Ibid., 28 de septiembre de 1932, p. 3; «Homenaje a Luis Bello-Comida íntima-El ministro de Gobernación a Madrid», La Vanguardia, 27 de septiembre de 1932, p. 29. 
Una vez concluido el periplo del presidente del Consejo, Tarradellas recibió la felicitación de la prensa por cómo había organizado su estancia en Barcelona. Él, a su vez, pidió que hicieran constar su agradecimiento a las entidades y corporaciones y al pueblo en general por la colaboración que había prestado en todos los actos (60). Otro éxito más en su meteórica carrera política.

\section{LA TRASLACIÓN DE LA EXPERIENCIA PROFESIONAL A LA POLÍTICA}

Josep Tarradellas trasladó a la política la experiencia profesional adquirida durante su progreso como viajante de comercio desde su inicio como tal a partir de 1923 y que durante el periodo republicano, pese a sus cargos políticos, no abandonó. Su capacidad organizativa, de trato y representatividad aplicada a las necesidades que requería la Generalitat provisional de Macià en el momento de su estructuración le convirtieron en una figura indispensable, junto con Amadeu Hurtado, en ese periodo inicial. En este sentido, la actuación de Tarradellas moviendo los hilos de la campaña de propaganda alrededor del Estatuto catalán es tan solo un ejemplo del papel que este jugó como secretario particular de Macià y consejero de Gobernación.

Al comenzar la Segunda República el nuevo gobierno catalán provisionalnecesitaba proyectar una, $s u$, imagen en un momento de auge comunicativo y de relación entre los líderes y las masas para asentar su poder político. En relación con este aspecto cabe destacar lo que significó para Tarradellas estar en todo momento al lado de un hombre, Macià, que basó gran parte de su popularidad en la imagen (61). Este elemento fue clave años después para cuando ejerció de presidente de la Generalitat en el exilio y se vio necesitado de aparentar, de representar, un poder que no tenía.

En segundo lugar, el relevo y la asunción de competencias propias o atribuibles a la consejería de Gobernación por parte de Tarradellas en detrimento de Joan Casanovas, a quien sustituyó a finales de 1931, afectaron su relación personal. A lo que cabría sumar, de nuevo, la sustitución de Casanovas por Tarradellas como consejero primero del gobierno Companys a finales de setiembre de 1936, ya comenzada la Guerra Civil española. Sin duda, una y otra vez «Tarra» se esforzó para demostrar que podía hacerlo mejor que su predecesor y eso deterioró su relación posterior. Precisamente, Casanovas, bandeado del poder, fue uno de los impulsores del complot que pretendió sustituir a Companys al frente de la Generalitat en octubre de 1936 (62).

Finalmente -e igual que los anteriores aspectos quedan apenas apuntados puesto que el espacio limita una argumentación en profundidad y no es objeto

(60) CONTRERAS (2008): 184-188; HuRTADO (2011): 697.

(61) UCELAY-DA CAL $(1979 ; 1983)$.

(62) UCELAY-da Cal, GonZalez (2012). 
de este artículo-, la participación de Tarradellas como parte activa en los entresijos de la elaboración, aprobación y ejecución del Estatuto de autonomía de Cataluña de 1932 fueron una experiencia que le enfrentó con quien sería a la postre su sucesor en el cargo de presidente, Jordi Pujol, cuando se discutió el Estatuto de autonomía de 1979. El presidente retornado del exilio sabía las dificultades a sortear porque había recorrido el camino antes, pero al inicio de los años ochenta del siglo pasado la Segunda República quedaba lejos y aquello que todavía coleaba se quería olvidar (63).

\section{BIBLIOGRAFÍA}

Albert Barris, M. (1939). Memorias inéditas. Montpellier: Archivo particular de sus descendientes.

Alcalá-Zamora, N. (2012). La victoria republicana. Los diarios robados del presidente de la Segunda República. 1930-1931. El derrumbe de la monarquía y el triunfo de una revolución pacífica. Madrid: La Esfera de los Libros.

Alquézar, R. (ed.) (2001). Esquerra Republicana de Catalunya, 70 anys d'història (1931-2011). Barcelona: Columna.

Arbós, A. (1988). Tarradellas. La consciència d'un poble. Barcelona: Ed. Grijalbo

Arrufat, R. (2007). Macià: la trajectòria política d'una figura excepcional en un poble que ha perdut la fe. Juneda: Fonoll.

Aymamí, L. (1932). Ambels braçosoberts. . . : reportatge de l'Estatut. Barcelona: Políglota.

Azaña, M. (2007). Obras completas. Vol. 6. Madrid: Servicio Central de Publicaciones, Ministerio de la Presidencia.

Bonamusa, F. (ed.) (2008). L'obra de govern de Josep Tarradellas (1936-1977): I Simposi d'Història sobre Josep Tarradellas. Lleida: Pagès editors.

Bricall, J. M. (1977). Política econòmica de la Generalitat (1936-1939). Barcelona: Edicions 62.

- (2003). Memoria de un silencio, el gobierno Tarradellas (1977-1980). Barcelona: Plaza\&Janés.

Casanelles, J. (1991). Joan Casanelles i Ibarz (1904-1986). Memòries i biografia, edición de Joan B. Culla i Clarà. Barcelona: Ajuntament de Barcelona.

Casanovas Casacuberta, J. (1996). Joan Casanovas i Maristany, president del Parlament de Catalunya. Barcelona: Publicacions de l'Abadia de Montserrat.

Casassas, J. (ed.) (2003). Josep Tarradellas, o, La reivindicació de la memòria (18991988). Lleida - Barcelona: Pagès editors - Diputació de Barcelona.

Conte, J. (2011). Tarradellas, testigo de España: el exilio y la Transación a través de los archivos privados de uno de los políticos mejor informados de su tiempo. Barcelona: Destino.

(63) BRICALL (2003). 
Contreras, J. (2008). Azaña y Cataluña. Historia de un desencuentro. Barcelona: Edhasa. Culla Clarà, J. (1981). Del republicanisme català al catalanisme d'esquerres (19221931). L'Avenç, 37, 33-37.

Duran, L. (2003). Josep Tarradellas, jove nacionalista. En Cassassas, J. (ed.) Josep Tarradellas o La reivindicació de la memòria (1899-1988). Lleida: Pagès editors.

Esculies, J. (2012a). Josep Tarradellas (1899-1936). Delsorígens a la República. Barcelona: EdicionsDau.

- (2012b). Josep Tarradellas (1899-1936). Dels origens a la Guerra Civil [tesis doctoral]. Universitat Pompeu Fabra.

(2013). El nacionalismo radical catalán (1913-1923). Spagna Contemporanea, $43,7-28$.

- (2014). La Falç (1918-1939). Història d'una joventut del nacionalisme radical català. Afers, 78, (29), 495-523.

(2015). Josep Andreu Abelló. Els clarobscurs del catalanisme. Barcelona: Edicions de 1984.

Febrés, X. (1985). Diàlegs a Barcelona. Josep Tarradellas i Antoni Gutiérrez Díaz. Barcelona: Ajuntament de Barcelona - Ed. Laia.

Gabriel, P. (2000). Francesc Macià y la contemporaneidad catalana de Don Niceto Alcalá-Zamora. En Casas Sánchez, J. L. y Durán Alcalá, F. (2000). V Jornadas Niceto Alcalá-Zamora y sus contemporáneos (pp. 13-42). Patronato Niceto Alcalá-Zamora y Torres: Diputación de Córdoba.

González Vilalta, A. (2003). La Oficina de la Generalitat de Catalunya en Madrid (1931-1933), al servicio de los diputados constituyentes. Revista de las Cortes Generales, 58, 110-127.

- (2006). La irrupció de la dona en el catalanisme (1931-1936). Barcelona: Publicacions de l'Abadia de Montserrat.

Hurtado, A. (2011) [1956, 1958, 1967]. Quaranta anys d'advocat. Història del meutemps (1894-1936). Barcelona: Edicions 62.

Ivern, M. D. (1988). Esquerra Republicana de Catalunya. Vol. I. Barcelona: Publicacions de l'Abadia de Montserrat.

- (1989). Esquerra Republicana de Catalunya. Vol. II. Barcelona: Publicacions de l'Abadia de Montserrat.

Jackson, Kenneth, T. (ed. ) (2010). The Encyclopedia of New York. Nueva York: Yale UniversityPress. Disponible en: http://dx. doi. org/10. 1126/science. 327. 5963. 269-a

Lladonosa, M. (1988). Catalanisme i moviment obrer; el CADCI entre 1903 i 1923. Barcelona: Publicacions de l'Abadia de Montserrat.

Llates, R. (1969). 30 anys de vida catalana. Barcelona: Editorial Aedos.

LluhíVallescà, J. (1932). Vermut popular d'homenatge a en Josep Tarradellas. 10 de julio, Documento transcrito, Fondo Gobierno Macià, Archivo Montserrat TarradellasMacià, Poblet.

Martín Berbois, J. L. (2013). Ignora des peròdesitjades: la dona política durant les eleccions de la Segona República a Catalunya. Barcelona: A Contra Vent.

Nevius, M. y Nevius, J. (2009). Inside the Apple. A Streetwise History of New York City. Nueva York: Free Press. 
Palau, E. (1932). Josep Tarradelles i els periodistas. El Reporter. Publicació del Centre de Reporters de Barcelona, 3, julio, sp.

Pla, J. (1996). Madrid-L'adveniment de la República. Madrid: Destino.

Peña González, J. (2002). Alcalá Zamora. Barcelona: Ariel.

Poblet, J. M. (1976). Història de l'Esquerra Republicana a Catalunya. Barcelona: Dopesa.

Porcel, B. (1977). Josep Tarradellas, president de la Generalitat. Barcelona: Ediciones AC.

Rivas Cherif, C. de (1961). Retrato de un desconocido: vida de Manuel Azaña. México: Oasis.

Rocarol, J. (1999). Memòries de Josep Rocarol i Faura (1882-1961). Barcelona: Ed. Hacer.

Roig i Rosich, J. M. (1978). L'Estatut de Catalunya a les Corts Constituents. Barcelona: Curial.

- (1992). La dictadura de Primo de Rivera a Catalunya: un assaig de repressió cultural. Barcelona: Publicacions de l'Abadia de Montserrat.

Sardà, Z. (1989). Francesc Maciàvist per la sevafilla Maria. Barcelona: Destino.

Sebag Montefiore, S. (2007). Young Stalin. London: Phoenix.

Sentís, C. (2002). I de sobte, Tarradellas. Barcelona. La Campana.

Sobrequés, J. (1988). El Restabliment de la Generalitat i el retorn del president Tarradellas. Barcelona: Barcanova.

Soler Serrano, J, (1977). Conversaciones con Tarradellas. Madrid: Sedmay.

Tarradellas, J. (1998). Dos mesos i mig al Departament de Cultura. Poblet: Arxiu Montserrat Tarradellas i Macià.

— (1999). Ja sóc aquí: record d'unretorn. Barcelona: Planeta.

(2007). La Indústria de Guerra a Catalunya (1936-1939). L'obra de la Comissió, creada per la Generalitat i el seu report d'actuació. Lleida: Pagès editors.

Ucelay-Da Cal,E. (1977). La formació d'Esquerra Republicana de Catalunya. L'Avenç, 4, 59-67.

- (1979). The Strategies of Separation and Revolution of Catalan Radical Nationalism (1919-1923)[Tesis doctoral]. Columbia University.

- (1982). La Catalunya populista. Imatge, cultura i política en l'etapa republicana (1931-1939). Barcelona: La Magrana.

- (1983). El nacionalisme radical català i la resistència a la Dictadura de Primo de Rivera (1923-1931) [Tesis doctoral]. Universitat Autònoma de Barcelona.

- (1984). Francesc Macià. Una vida en imatges. Barcelona: Generalitat de Catalunya.

- (1987). Joventut i nacionalisme radical català, 1910-1987. En Ibid., (dir.): La Joventut a Catalunya al segle XX. Vol. I. Barcelona:Diputació de Barcelona.

Ucelay-Da Cal, E. y González Vilalta, A. (eds.) (2012). Contra Companys, 1936. La frustración nacionalista ante la Revolución. Valencia: Publicacions de la Universitat de València.

Udina, E. (1978). Tarradellas. L'aventura d'una fidelitat. Barcelona: Edicions 62.

Viusà, M. (1968). Francesc Macià. París: Som-Associació Catalònia. 